\title{
A CLASS OF STABILITY CRITERIA FOR HILL'S EQUATION*
}

BY HARRY HOCHSTADT (Polytechnic Institute of Brooklyn)

The purpose of this note is to prove the following theorem: A sufficient condition for the boundedness of all solutions of

$$
y^{\prime \prime}+p(t) y=0,
$$

where $p(t)$ is an even, positive, differentiable function of period $T$, is that

$k \pi \leq \int_{0}^{T}[p(t)]^{1 / 2} d t-\frac{1}{4} \int_{0}^{T}\left|\frac{p^{\prime}(t)}{p(t)}\right| d t \leq \int_{0}^{T}[p(t)]^{1 / 2} d t+\frac{1}{4} \int_{0}^{T}\left|\frac{p^{\prime}(t)}{p(t)}\right| d t \leq(k+1) \pi$ for some integer $k \geq 0$.

The proof of the theorem is based on a method developed in connection with a general analysis of the Sturm-Liouville spectrum [1]. There it is shown that the even and odd solutions of the differential equation, which are denoted by $y_{1}$ and $y_{2}$ respectively, can be represented as

$$
\begin{array}{ll}
y_{1}=A_{1}(t) \cos \phi_{1}(t), & y_{2}=A_{2}(t) \sin \phi_{2}(t) \\
y_{1}^{\prime}=-[p(t)]^{1 / 2} A_{1}(t) \sin \phi_{1}(t), & y_{2}^{\prime}=[p(t)]^{1 / 2} A_{2}(t) \cos \phi_{2}(t) .
\end{array}
$$

A direct calculation shows that the functions $\phi_{i}(t), A_{i}(t)$ must satisfy the differential equations

$$
\begin{aligned}
& \phi_{1}^{\prime}=[p(t)]^{1 / 2}-\frac{1}{4} \frac{p^{\prime}(t)}{p(t)} \sin 2 \phi_{1}, \\
& \phi_{2}^{\prime}=[p(t)]^{1 / 2}+\frac{1}{4} \frac{p^{\prime}(t)}{p(t)} \sin 2 \phi_{2}, \\
& A_{1}^{\prime}=-A \frac{p^{\prime}(t)}{2 p(t)}\left(\sin \phi_{1}\right)^{2}, \\
& A_{2}^{\prime}=-A \frac{p^{\prime}(t)}{2 p(t)}\left(\cos \phi_{2}\right)^{2},
\end{aligned}
$$

and

$$
A_{1}(0)=A_{2}(0)=1, \quad \phi_{1}(0)=\phi_{2}(0)=0 .
$$

If we consider a function $p(t)$ of the form

$$
p(t)=\lambda+\Psi(t),
$$

where $\lambda$ is a parameter we obtain periodic solutions if and only if $\lambda$ belongs to a discrete set of eigenvalues arranged in the following ascending sequence

$$
-\infty<\lambda_{0}<\lambda_{1}^{\prime} \leq \lambda_{2}^{\prime}<\lambda_{1} \leq \lambda_{2}<\lambda_{3}^{\prime} \leq \lambda_{4}^{\prime}<\lambda_{3} \leq \lambda_{4} \cdots
$$

(see [2]). If $\lambda$ is equal to some $\lambda_{i}$ at least one solution of the equation has period $T$, but 
if $\lambda$ is equal to some $\lambda_{i}^{\prime}$ at least one solution has period $2 T$. One can easily show [1] that the $\lambda_{i}$ must satisfy the conditions

$$
\phi_{1}(T)=2 k \pi
$$

if the corresponding periodic solution of the differential equation is even and

$$
\phi_{2}(T)=2 k \pi
$$

if the solution is odd. Similarly the $\lambda_{i}^{\prime}$ satisfy

$$
\begin{aligned}
& \phi_{1}(T)=(2 k+1) \pi, \\
& \phi_{2}(T)=(2 k+1) \pi,
\end{aligned}
$$

corresponding to even and odd solutions respectively.

Whenever $\lambda$ lies in one of the intervals

$$
\left(\lambda_{0}, \lambda_{1}^{\prime}\right), \quad\left(\lambda_{2}^{\prime}, \lambda_{1}\right), \quad\left(\lambda_{2}, \lambda_{3}^{\prime}\right), \cdots
$$

both solutions of the differential equation are bounded for all real $t[2]$. This can only happen if both $\phi_{i}(T)$ satisfy the inequalities

$$
k \pi \leq \phi_{i}(T) \leq(k+1) \pi
$$

for some integer $k \geq 0$. Since

$$
\phi_{i}(T)=\int_{0}^{T}[p(t)]^{1 / 2} d t \mp \frac{1}{4} \int_{0}^{T} \frac{p^{\prime}(t)}{p(t)} \sin 2 \phi_{i} d t, \quad\left\{i=\frac{1}{2}\right.
$$

the conclusion of the theorem follows from elementary considerations. This theorem generalizes a stability criterion proved in an earlier publication [3].

\section{REFERENCES}

[1] H. Hochstadt, Asymptotic estimates for the Sturm-Liouville spectrum, to be published, Comm. Pure and Appl. Math.

[2] E. A. Coddington, N. Levinson, Theory of ordinary differential equations, McGraw-Hill, New York, 1955, Chapter 8.

[3] H. Hochstadt, A stability criterion for Hill's equation, to be published, Proc. Amer. Math. Soc. 